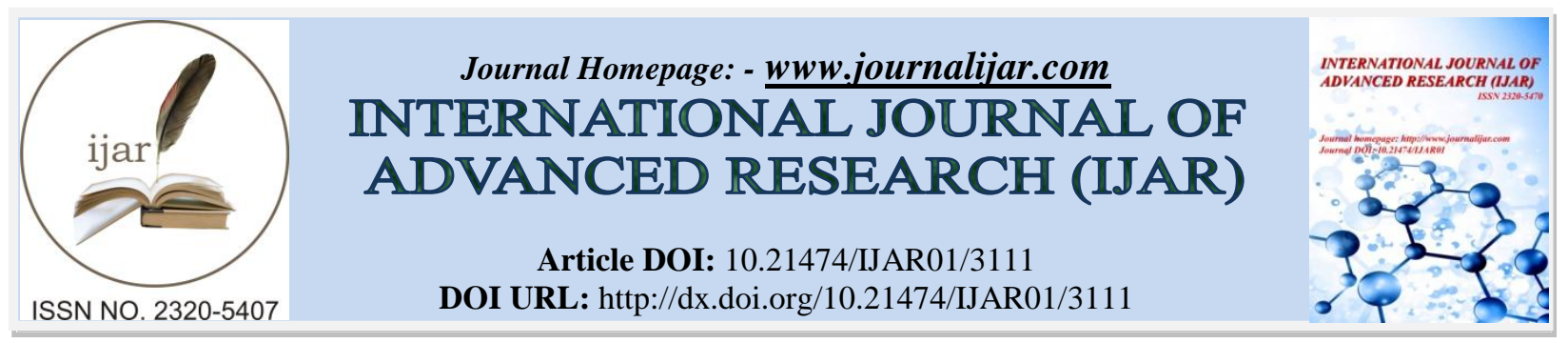

RESEARCH ARTICLE

\title{
HEAVY IONS REACTIONS WITH TWO NUCLEON TRANSFER.
}

\author{
M. Zaky and A. Kh. Khawaldeh.
}

Physics Department, Faculty of Science, Cairo University, Cairo, Egypt.

\section{Manuscript Info}

Manuscript History

Received: 03 December 2016

Final Accepted: 06 January 2017

Published: February 2017

\begin{abstract}
The ${ }^{64} \mathrm{Ni}\left({ }^{16} \mathrm{O},{ }^{14} \mathrm{C}\right){ }^{66} \mathrm{Zn}$ two proton pickup reactions has been studied , at $56 \mathrm{Mev}$ of ${ }^{16} \mathrm{O}$ projectile energy, the differential cross section have been evaluated in the frame work of the exact finite-range distorted wave Born approximation using folding model for the real part of optical nuclear potential. The calculated angular distributions are fitted with the experimental data .
\end{abstract}

Copy Right, IJAR, 2017,. All rights reserved.

\section{Introduction:-}

The two nucleon transfer reactions have been taken on great importance , because they are very sensitive to nuclear spectroscopy, heavy ion transfer reactions are valuable tools for getting precise spectroscopic information's [1, 2]. The right framework in which the reaction be treated is Distorted Wave Born Approximation ( DWBA), or Coupled Channel Born approximation (CCBA) methods [3,4] with inclusion the nuclear recoil. Two nucleon transfer reactions induced by heavy ions had been widely considered [5] by using DWBA with nucleon - nucleon interactions which include both attraction and repulsion.

In the present study, the differential cross section of heavy ion reaction with two proton pickup reaction have been calculated in The ${ }^{64} \mathrm{Ni}\left({ }^{16} \mathrm{O},{ }^{14} \mathrm{C}\right){ }^{66} \mathrm{Zn}$ reaction at $56 \mathrm{Mev}$ of $16 \mathrm{O}$ projectile energy, analysis concentrated on g.s. ,first $2^{+}$,first $3^{-}$excited levels. The ${ }^{64} \mathrm{Ni}\left({ }^{16} \mathrm{O},{ }^{14} \mathrm{C}\right){ }^{66} \mathrm{Zn}$ are studied by Mermaz [7] by using coupled channel Born approximations and distorted wave born approximation calculations and he conclude that the relative intensities among various states are better reproduced by CCBA than DWBA calculations, the present work is like Memaz study using DWBA but the optical potential model was taken to be folded for the real part of optical potential, the phenomenological optical potential are often successfully used to describe the heavy ion elastic scattering data ,the use of folding model allows us to predict potential and eliminate the ambiguities which appear with the phenomenological potential. Folding model gives better interpretation as a basic physical ingredients the nuclear densities folded with a nuclear interaction in correct way. The calculated angular distributions are fitted with the experimental data.

\section{The differential cross section of heavy ion reaction with nucleon}

Transfer:

The differential cross section of heavy-ion reaction with two nucleons pickup

$\mathrm{A}(\mathrm{b}, \mathrm{a}) \mathrm{B}$ where $\mathrm{A}$ is the target $\mathrm{B}$ is the residual nucleus, $\mathrm{b}$ is the projectile $\mathrm{A}=\mathrm{B}+\mathrm{c}$, and $\mathrm{a}=\mathrm{b}+\mathrm{c}$, and the $\mathrm{c}$ is the two - proton cluster, is given by the expression:

$\frac{d \sigma}{d \Omega}=\frac{m_{a B} m_{b A}}{\left(2 \pi \hbar^{2}\right)^{2}} \frac{K_{a B}}{K_{b A}} \frac{1}{2 S_{b}+1} \sum_{L M}\left|T_{f i}\right|^{2}$ 
Where $m_{b B}, m_{b A}$ are the reduced masses of pair (a, B ), ( b , A ) respectively, and the matrix element $T_{f i}$ represent the DWBA transition amplitude that has the form :

Where:

$$
T_{f i}=\sum_{L_{c B} L_{c b} N_{c B} N_{c b}} B_{c B} \beta_{L M}
$$

$$
\begin{gathered}
\beta_{L M}=\frac{(-1)^{L}}{\sqrt{2 L+1}} \int d r_{b A} d r_{a B} X^{*}\left(r_{b A}\right) f_{L M}\left(\boldsymbol{r}_{c b}, \boldsymbol{r}_{c B}\right) \\
f_{L M}=\sum_{\Lambda \bar{\Lambda}}(-1)^{L_{c B}+\bar{\Lambda}}\left\{L_{c B} \Lambda L_{c b} \mid L M>\Phi_{\Lambda}^{*}\left(r_{c B}\right) V\left(\boldsymbol{r}_{c b}\right) \Phi_{\bar{\Lambda}}\left(\boldsymbol{r}_{c b}\right)\right. \\
B_{c B}=i^{L} \sum_{L_{c}}\left(\frac{2 J_{a}+1}{2 L_{c}+1}\right)^{\frac{3}{2}}(-1)^{L_{c B}+L_{c}-J_{B}} U\left(I_{B} L_{c B} J_{a} L_{c b} ; L_{C} L\right) A_{c B}^{*} A_{c b}
\end{gathered}
$$

The dependence on kinematics and the reaction mechanism is contained in the factor $\beta_{L M}$, where $f_{L M}\left(r_{c B}, r_{c B}\right)$ is the finite-range form factor. The spectroscopic dependence lies in the factor $B_{C B}$, and the two spectroscopic amplitude $A_{c B}, A_{c b}$ contained therein.and $V(r)$ is nuclear optical model potential in form of Wood-saxon, this form is given as :

$$
V(r)=\frac{-V_{0}}{1+\exp \left\{\frac{r-R_{V}}{a_{v}}\right\}}+\frac{-W_{0}}{1+\exp \left\{\frac{r-R_{w}}{a_{w}}\right\}}
$$

Where, the parameters $V_{0}, \mathrm{R}_{\mathrm{v}}$ and $\mathrm{a}_{\mathrm{v}}$, are the strength, radius and diffuseness of the real potential, while the parameters $W_{0}, \mathrm{R}_{\mathrm{w}}$ and $\mathrm{a}_{\mathrm{w}}$, are the strength ,radius and diffuseness of the imaginary part which are determined by fitting scattering reaction of the corresponding interaction of two heavy ions.

\section{Numerical calculations and result and discussion and conclusion:-}

The differential cross section has been numerically carried out for ${ }^{64} \mathrm{Ni}\left({ }^{16} \mathrm{O},{ }^{14} \mathrm{C}\right){ }^{66} \mathrm{Zn}$ reaction at $56 \mathrm{Mev}$, in the frame work of the exact finite-range distorted wave Born approximation , and the real double -folded potential used in an optical model analysis of elastic scattering data. The potentials were calculated by convoluting the M3Y effective nucleon - nucleon interaction[8], the necessary parameters of the optical potential are shown in table (1). here the results obtained for the differential cross section are shown in figure(1), figure(2) and figure(3).

Table ( 1 ) : Optical potential parameters used in DWBA calculations

\begin{tabular}{|c|c|c|c|c|c|c|}
\hline Reaction & $\begin{array}{c}\mathrm{E}_{\mathrm{lab}} \\
(\mathrm{Mev})\end{array}$ & $\begin{array}{c}\mathrm{W}_{0} \\
(\mathrm{Mev})\end{array}$ & $\mathrm{a}_{\mathrm{w}}$ & $\mathrm{R}_{\mathrm{w}}$ & $\mathrm{r}_{\mathrm{c}}$ & Ref. \\
\hline${ }^{16} \mathrm{O}+{ }^{64} \mathrm{Ni}$ & $56 \mathrm{Mev}$ & 20 & 0.283 & 1.311 & 1.25 & 7 \\
\hline
\end{tabular}




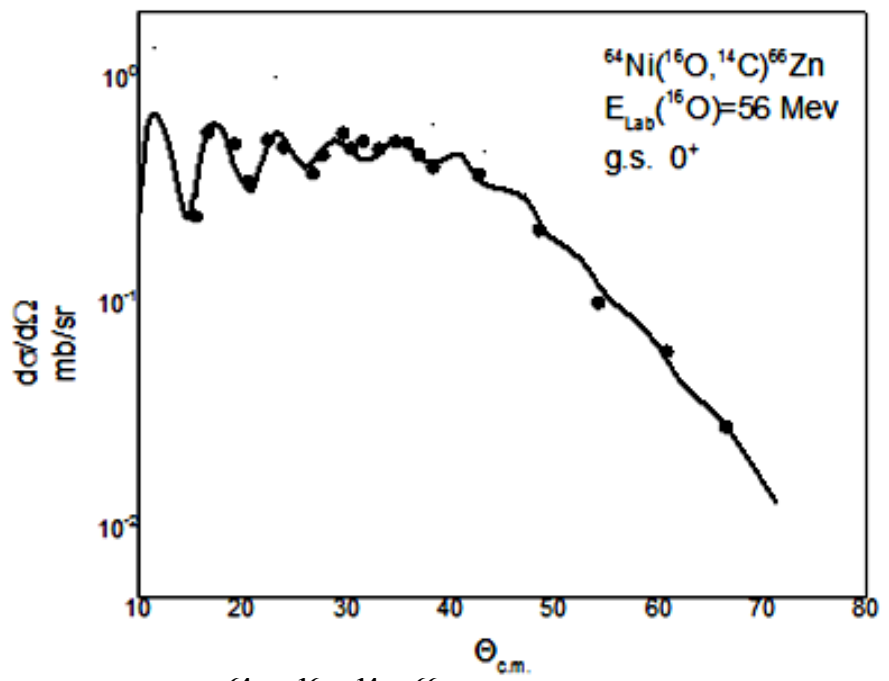

Figure 1:- The differential cross section ${ }^{\mathbf{6 4}} \mathbf{N i}\left({ }^{\mathbf{1 6}} \mathbf{O},{ }^{\mathbf{1 4}} \mathbf{C}\right){ }^{\mathbf{6 6}} \mathbf{Z n}$ reaction at $56 \mathrm{Mev}$ incident energy leading to ${ }^{66} \mathrm{Zn}$ ground state .The solid curve is the present calculation and the dots are the experiment data taken from refernce (9).

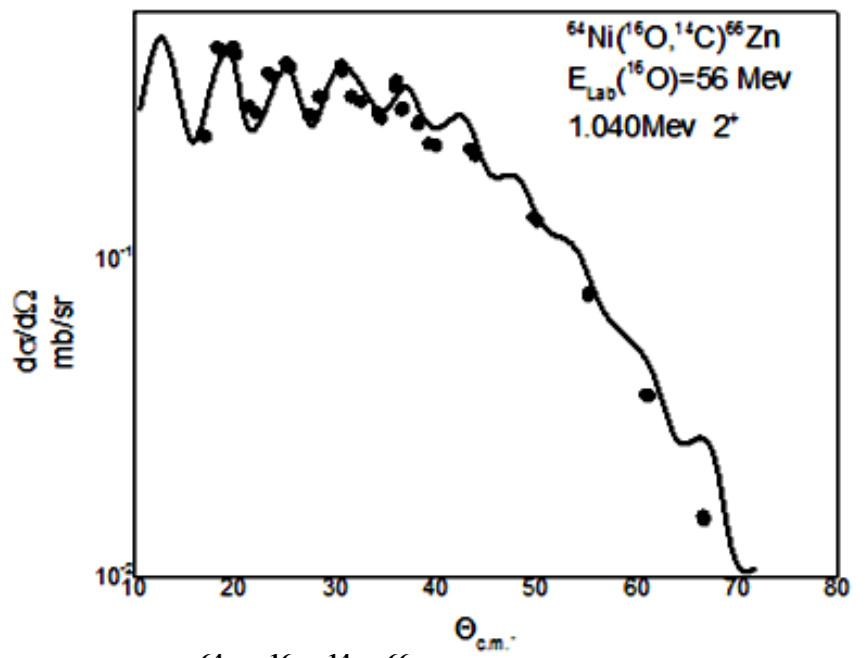

Figure2:- The differential cross section ${ }^{64} \mathrm{Ni}\left({ }^{16} \mathrm{O},{ }^{14} \mathrm{C}\right){ }^{66} \mathrm{Zn}$ reaction at $56 \mathrm{Mev}$ incident energy leading to $66 \mathrm{Zn}$ at $1.040 \mathrm{Mev} 2^{+}$state .The solidcurve is the present calculation and the dots are the experiment data taken from refernce (9) 


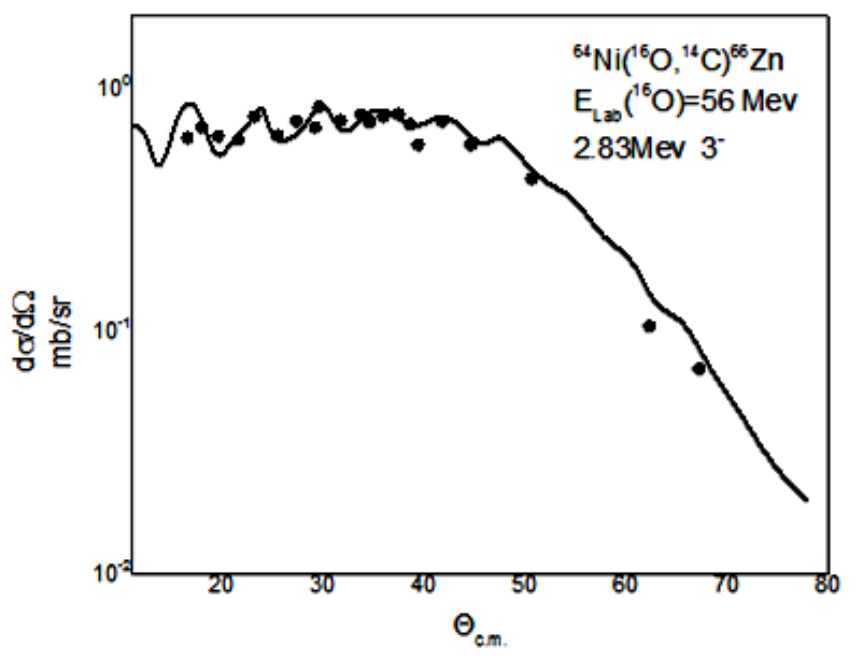

Figure3:- The differential cross section $64 \mathrm{Ni}(16 \mathrm{O}, 14 \mathrm{C}) 66 \mathrm{Zn}$ reaction at $56 \mathrm{Mev}$ incident energy leading to $66 \mathrm{Zn}$ at $2.830 \mathrm{Mev} 3$ state. The solid curve is the present calculation and the dots are the experiment data taken from refernce (9).

The shape of all angular distributions are in a good agreement with the experimantal data except that the angular distribution for transition $2^{+}$states, it seem to be several degrees forward of the DWBA curve. In conclusion in this study despite the better shape of angular distributions with a little difference in the shape of angular distributions clalculated by DWBA using the double-folding model for the real part of optical potential and the previous calculations without using the folded model ,the CCBA calculations ( 8 )which include multiple proccesses are more suitable than DWBA they give better a greement with experimantal data, and the double-folding potential are found to be appropriate to reproduce the cross-section and capable of producing realistic prediction of the angular distribution.

\section{References:-}

1. Anyas_Weiss, N., et al., Phys. Rep.C, 1974, vol. 12, p. 201.

2. Kahana, S. and Baltz, A.J., Adv. Nucl. Phys., 1977, vol. 9, p. 1.

3. Satchler, G.R., Direct Nuclear Reactions, Oxford: Oxford Univ. Press, 1983.

4. G.R. Satchler, Direct Nuclear Reactions, Oxford Science Publications, 1983

5. A.Osman : Part.Nucl.,4,209 (1972);N uavoCimento A,37,201 (1977) 39,138 (1977);42,330 (1977)

6. P.D. Kunz, DWUCK5, computer program, http://spot.colorado.edu/ kunz/DWBA.html

7. M.C. Mermaz, et al., Phys. Rev. C 20 (1979) 2130

8. Hasan Maridi ., Scattering of halo nuclei,LAP Lambert Academic (2013) ,pp.16-19

9. M.C.Lemaire, M.C. Mermaz, H. Sztark, and A. Cunsolo, Phys. Rev. C 10, 1103 (1974). 